\title{
Obituary
}

\section{Dr Robert George (Rob) Jones FRCPsych}

\section{Formerly Honorary Professor of Old Age Psychiatry, University of Nottingham}

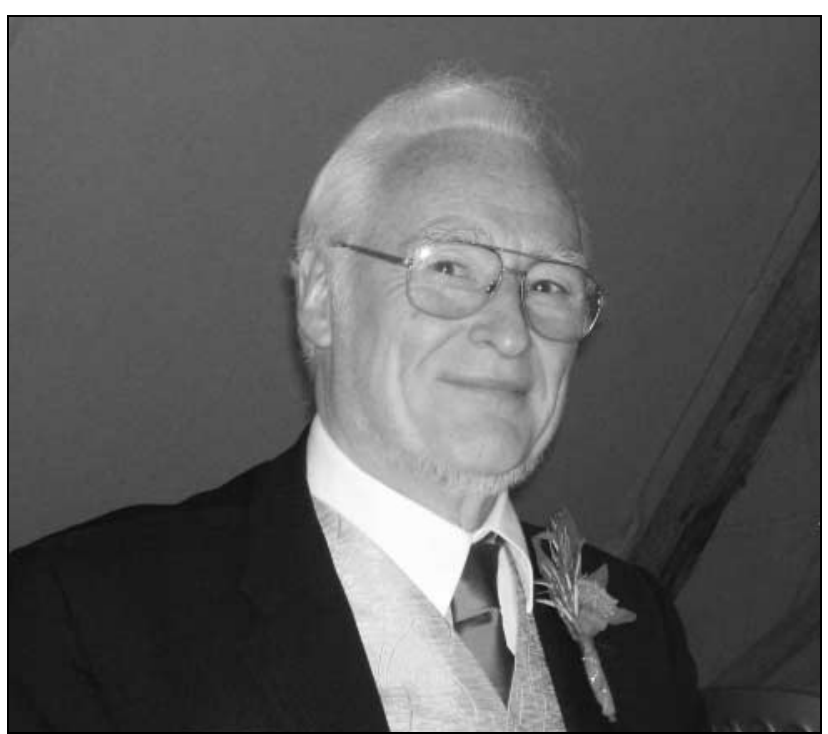

For over 30 years Rob Jones, who died recently at the age of 69, was a pillar of old age psychiatry in Nottingham and beyond. Qualifying in Manchester in 1970, he trained there in psychiatry and was particularly impressed with David Jolley's work in old age psychiatry. Following research with David Goldberg, he moved in 1980 to Nottingham as senior lecturer in psychiatry in the department of Health Care of the Elderly that Tom Arie had newly set up. This comprised physicians, psychiatrists and other health professions - Rob worked opposite John Bendall, the senior lecturer in medicine, and was a key figure in the ambitious 1-month combined attachment in old age medicine and psychiatry for all medical students. The novel joint department attracted wide interest, visitors and attached workers coming from home and abroad. Courses in psychogeriatrics sponsored by the British Council or the World Health Organization brought workers from more than 30 countries to Nottingham and Nottingham staff were often invited abroad. Rob also contributed to a British Council course in Warsaw.

Rob's research included studies of outcomes for care home residents and of community provision; he also participated in major national studies and published widely. He was involved in collaborations that have shaped the practice of old age psychiatry across the UK and internationally. These included the DOMINO-AD clinical trial, ${ }^{1}$ which has shown that continuing anti-dementia drugs in people with moderate to severe Alzheimer's disease is worthwhile and does reduce the likelihood of entering a care home at least for a few months.

His most important research was around the care and services for older people with dementia, including, with lan Rothera and others, a study of life expectancy after entering residential care. ${ }^{2}$ More recently, he provided the psychiatry input into the Medical Crises in Older People programme led by John Gladman. This documented just how many very elderly, frail and vulnerable people are admitted to hospital. ${ }^{3}$ His later work included studies of care for people with dementia living at home and at the time of his death he was involved in the NIHR Programme Grant PrAISED: Promoting Activity, Independence and Stability in Early Dementia, led by Rowan Harwood.

Rob was a busy clinician and led a district service for older people which served some of the most socially deprived parts of Nottingham, whenever possible taking services to people's own homes. He retired from clinical work in 2013 but continued to be a trustee of the local Radford Care Group, reflecting his passion for the well-being of people living at home, particularly those with dementia. He continued also to work for the university, heading the teaching programme in Health Care of the Elderly until his full retirement in October 2015. Of course, this was not the last we saw of him! He carried on with his research interests, as well as work on public involvement in dementia research - he organised the monthly Centre for Dementia seminars at the Institute of Mental Health. His contributions were recognised by the university with an honorary professorship.

Rob Jones was born in Paignton, Devon, into a family of proud Welsh ancestry. His father edited the local newspaper and this doubtlessly contributed to his insatiable lifelong interest in current affairs. At home he was permanently tuned into BBC Radio 4 and his colleagues were often deeply impressed by his detailed knowledge of world news and politics, to say nothing of lower league football (Torquay United in particular). In Manchester he met Diane and they married in 1971.

Rob was a man of warm and generous personality. He was regarded with respect and affection by all who knew him. His enthusiasm and commitment to the cause of older people was inspiring. For the last 20 years of his career he led academic old age psychiatry in Nottingham, as well as the Trent Dementia Research Network from 2004 until the establishment of the Nottingham Centre for Dementia in 2014. A particular mission was to keep us psychiatrists in close alliance with our colleagues in geriatric medicine, notably through the combined medical student programme. He was especially noted for bundles of papers in carrier bags, festoons of keys and lanyards at his neck, and his characteristic hearty laugh.

Notwithstanding his interest in people, he was quite private. For example, nobody he worked with was aware that Rob had been living with multiple sclerosis for 15 years. Indeed, he completed numerous half marathons during this time. He was definitely not one to complain. After he suffered a myocardial infarction in 2015, we were able to welcome him back to work. It is no surprise, therefore, that his sudden death on 23 May 2016 from a cerebellar haemorrhage came as a blow to all.

He leaves his wife Diane, and his children Haydn, Rhian, David and Siân, along with nine grandchildren.

Tom Dening and Tom Arie 


\section{References}

1 Jones R, Sheehan B, Phillips P, Juszczak E, Adams J, Baldwin A, et al. DOMINO-AD protocol: donepezil and memantine in moderate to severe Alzheimer's disease: a multicentre RCT. Trials 2009; 10: 57.

2 Rothera IC, Jones R, Harwood R, Avery AJ, Waite J. Survival in a cohort of social services placements in nursing and residential homes: factors associated with life expectancy and mortality. Public Health 2002; 116 $160-5$

3 Bradshaw LE, Goldberg SE, Lewis SA, Whittamore K, Gladman JR, Jones RG, et al. Six-month outcomes following an emergency hospital admission for older adults with co-morbid mental health problems indicate complexity of care needs. Age Ageing 2013; 42: 582-8.

doi: 10.1192/pb.bp.116.055632

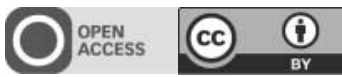

(C) 2017 The Authors. This is an open-access article published by the Royal College of Psychiatrists and distributed under the terms of the Creative Commons Attribution License (http://creativecommons.org/licenses/by/4.0), which permits unrestricted use, distribution, and reproduction in any medium, provided the original work is properly cited.

\section{Reviews}

\section{Mental Health in the Digital Age: Grave Dangers, Great Promise}

Edited by Elias Aboujaoude and Vladan Starcevic Oxford University Press, 2015, E29.58, pb, 302 pp. ISBN: 9780199380183

Technology has always been a double-edged sword: there are associated risks and benefits. As a practising psychiatrist I increasingly rely on technology at work, using next-generation electronic medical records and at times recommending appropriate smartphone-based applications as additional therapy for my patients.

In contrast to numerous other titles about technology and its impact on healthcare - which have emerged as a result of the massive technical advances in the past decade - Mental Health in the Digital Age does not focus only on the benefits of the use of technology in mental healthcare. It offers a timely balanced perspective by also providing an in-depth analysis of the risks.

The risks highlighted in the book are not limited to addictive behaviours such as internet or gaming addiction, but also include cyberbullying and the increased risk of suicide due to pro-suicide websites and suicide pacts. Cyberbullying is perhaps one of the most common problems linked with the use of technology to date and it is not unusual for me and my team to see children and adolescents who refuse to go to school as a result of cyberbullying. Unlike conventional forms of bullying, cyberbullying implies the use of social networks and internetbased messaging services to harass an individual. This work examines not only the prevalence of the problem, but also the various prevention strategies available, such as having a specific academic curriculum to deal with the issue.

The authors review the existing literature comprehensively - referring also to current evidence - and look at the potential of technology across several areas of mental healthcare, including the provision of psychotherapy and the integration of patients' health records. They also discuss how recent advances - such as virtual reality - could in principle be a powerful tool in exposure therapy. As a team with an interest in e-health, my colleagues and I have been developing smartphone applications for various mental health disorders. The introduction of virtual reality technology means that we could perhaps tap on games and various other sensors and headset devices to create an interactive environment not just for psychotherapy but for other forms of interventions too.

This is a good guide for novices in e-health but equally a useful tool for the more experienced in this area. It would be helpful if a future edition included more detailed coverage of smartphone applications and their inherent risks and benefits a topic of concern not only for clinicians, but patients at large.

Melvyn W. B. Zhang, Associate Consultant Psychiatrist and Adjunct Research Scientist, Institute of Mental Health, Biomedical Institute for Global Health Research \& Technology and National University of Singapore; Singapore; email: ciezwm@nus.edu.sg doi: 10.1192/pb.bp.116.054650

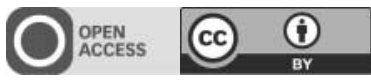

(C) 2017 The Author. This is an open-access article published by the Royal College of Psychiatrists and distributed under the terms of the Creative Commons Attribution License (http://creativecommons.org/licenses/by/4.0), which permits unrestricted use, distribution, and reproduction in any medium, provided the original work is properly cited.

\section{MCQs in Psychiatry for Medical Students}

By John Lally and John Tully. RCPsych Publications, 2016, $€ 18.00$ ( $£ 16.20$ for College members), pb, $224 \mathrm{pp}$. ISBN: 9781909726482

Love them or loathe them, most medical student written examinations now take the form of multiple choice questions (MCQs). Some medical educators dislike this assessment style, suggesting it encourages students to learn isolated facts in a superficial way. Yet, undeniably, MCQs provide an objective, time-efficient manner of evaluation.

MCQs in Psychiatry for Medical Students is a valuable resource for medical students undertaking their psychiatry rotations. It includes MCQs and extended matching items grouped into chapters concerned either with a type of disorder - for example, psychotic disorders and alcohol and substance misuse disorders - or another important aspect of psychiatry, such as physical health, pharmacological treatments, psychology and psychotherapy. 